\title{
Nonlinear fractional differential equations with nonlocal integral boundary conditions
}

\author{
Suli Liu', Huilai Li’ ${ }^{*}$ and Qun Dai
}

\section{"Correspondence:}

lihuilai@jlu.edu.cn

${ }^{1}$ School of Mathematics, Jilin

University, Changchun, 130012,

P.R. China

Full list of author information is

available at the end of the article

\section{空 Springer}

\begin{abstract}
This paper concerns the boundary value problem of a class of fractional differential equations involving the Riemann-Liouville fractional derivative with nonlocal integral boundary conditions. By using the properties of the Green's function and the monotone iteration technique, one shows the existence of positive solutions and constructs two successively iterative sequences to approximate the solutions, especially numerically simulates the conclusion by an example.
\end{abstract}

Keywords: fractional differential equation; integral boundary condition; iterative sequence

\section{Introduction}

In this paper, we investigate a class of nonlinear fractional differential equations with nonlocal integral boundary value conditions of the form

$$
\left\{\begin{array}{l}
D_{0+}^{\alpha} u(t)+f(t, u(t))=0, \quad 0<t<1, \\
u(0)=u^{\prime}(0)=u^{\prime \prime}(0)=0, \\
u(1)=\lambda I_{0+}^{\beta} u(\eta)=\lambda \int_{0}^{\eta} \frac{(\eta-s)^{\beta-1} u(s)}{\Gamma(\beta)} \mathrm{d} s,
\end{array}\right.
$$

where $3<\alpha \leq 4,0<\eta \leq 1, \lambda, \beta>0,0 \leq \frac{\lambda \Gamma(\alpha) \eta^{\alpha+\beta-1}}{\Gamma(\alpha+\beta)}<1$, and $D_{0+}^{\alpha}$ is the standard RiemannLiouville differential operator.

It is well known that fractional order models are more realistic and practical than the classical integer order models (see, e.g., [1-7]). As a result, many mathematicians show strong interest in fractional differential equations and many wonderful results have been obtained. The techniques of nonlinear analysis, as the main method to deal with the problems of nonlinear fractional differential equations, plays an essential role in the research of this field, such as establishing the existence and the uniqueness or the multiplicity of solutions to nonlinear fractional differential equations (see, e.g., [8-16] and the references therein). Among these techniques, the monotone iteration scheme is an interesting and effective way to investigate the existence of solutions to nonlinear fractional problems (see, e.g., $[17-19])$.

Ahmad and Nieto [20] studied the existence and the uniqueness of solutions to the following nonlinear fractional integro-differential equation:

$$
\left\{\begin{array}{l}
D_{0+}^{\alpha} u(t)=f(t, u(t),(\phi u)(t),(\psi u)(t)), \quad t \in[0, T], \\
D_{0+}^{\alpha-2} u\left(0^{+}\right)=0, \quad D_{0+}^{\alpha-1} u\left(0^{+}\right)=v I_{0+}^{\alpha-1} u(\eta),
\end{array}\right.
$$

(c) 2015 Liu et al. This article is distributed under the terms of the Creative Commons Attribution 4.0 International License (http://creativecommons.org/licenses/by/4.0/), which permits unrestricted use, distribution, and reproduction in any medium, provided you give appropriate credit to the original author(s) and the source, provide a link to the Creative Commons license, and indicate if changes were made. 
where $1<\alpha \leq 2,0<\eta<T, v$ is a constant, $f:[0, T] \times \mathbb{R} \times \mathbb{R} \times \mathbb{R} \rightarrow \mathbb{R}$ is continuous, and

$$
(\phi x)(t)=\int_{0}^{t} \gamma(t, s) x(s) \mathrm{d} s, \quad(\psi x)(t)=\int_{0}^{t} \delta(t, s) x(s) \mathrm{d} s
$$

with $\gamma$ and $\delta$ being continuous functions on $[0, T] \times[0, T]$. In [21], Ahmad and Agarwal considered the existence and the uniqueness of solutions to a class of Caputo type fractional differential equation of order $q \in(n-1, n]$ with slit-strips type boundary conditions

$$
\left\{\begin{array}{l}
{ }^{\mathrm{c}} D^{q} u(t)=f(t, u(t)), \quad 0<t<1, \\
u(0)=u^{\prime}(0)=\cdots=u^{(n-2)}(0)=0, \\
u(\eta)=a \int_{0}^{\xi} u(s) \mathrm{d} s+b \int_{\zeta}^{1} u(s) \mathrm{d} s,
\end{array}\right.
$$

where $0<\xi<\eta<\zeta<1, a$ and $b$ are positive constants. In [22], the authors considered a nonlinear fractional boundary value problem on a half-line given by

$$
\left\{\begin{array}{l}
D_{0+}^{\alpha} u(t)+f\left(t, u(t), D_{0+}^{\alpha-1} u(t)\right)=0, \quad t>0 \\
u(0)=0, \quad D_{0+}^{\alpha-1} u(\infty)=\beta u(\xi)
\end{array}\right.
$$

where $1<\alpha \leq 2, \xi>0$. The positive extremal solutions and iterative schemes for approximating them were obtained by applying a monotone iterative method.

Zhang et al. [23] studied the existence of positive solutions to the following fractional boundary value problem:

$$
\left\{\begin{array}{l}
D_{0+}^{\alpha} u(t)+h(t) f(t, u(t))=0, \quad 0<t<1, \\
u(0)=u^{\prime}(0)=u^{\prime \prime}(0)=0, \\
u(1)=\lambda \int_{0}^{\eta} u(s) \mathrm{d} s
\end{array}\right.
$$

where $3<\alpha \leq 4,0<\eta \leq 1,0 \leq \frac{\lambda \eta^{\alpha}}{\alpha}<1$. They got some results as regards the existence of positive solutions by using the properties of the Green's function, the boundedness of $u_{0}$, and the fixed point index theory. Jiang et al. [24] studied the fractional boundary value problem (1.2); $h(t) f(u(t))$ and $\lambda \int_{0}^{\eta} u(s) \mathrm{d} s$ were replaced by $f(t, u(t))$ and $\int_{0}^{\eta} u(s) \mathrm{d} s$, respectively. The authors obtained the existence of positive solutions to the problem (1.2) by using the monotone iterative method.

Motivated by the works mentioned above, in this article we study the differential equations (1.1) by using the fixed point theorem for increasing operators on the order intervals. We not only obtain the existence of positive solutions, but we also establish two iterative sequences to approximate the solutions. It should be pointed out that our method is different from that in [25]. The first term of the iterative sequence may be taken as a constant function or a simple function.

This paper is arranged as follows. Some lemmas needed below are listed in Section 2. The existence of the positive solutions to the problem (1.1) is proved and two successively iterative sequences to approximate the solutions are constructed in Section 3. Finally, in Section 4, an example is given to numerically simulate our conclusion. 


\section{Some lemmas}

Lemma 2.1 Assume that $y(t) \in C([0,1])$, then the solution to boundary value problem

$$
\left\{\begin{array}{l}
D_{0+}^{\alpha} u(t)+y(t)=0, \quad 0<t<1, \\
u(0)=u^{\prime}(0)=u^{\prime \prime}(0)=0 \\
u(1)=\lambda I_{0+}^{\beta} u(\eta)=\lambda \int_{0}^{\eta} \frac{(\eta-s)^{\beta-1} u(s)}{\Gamma(\beta)} \mathrm{d} s
\end{array}\right.
$$

can be given by

$$
u(t)=\int_{0}^{1} G(t, s) y(s) \mathrm{d} s
$$

where

$$
G(t, s)= \begin{cases}\frac{-P \Gamma(\alpha+\beta)(t-s)^{\alpha-1}+\Gamma(\alpha+\beta)(1-s)^{\alpha-1} t^{\alpha-1}-\Gamma(\alpha) \lambda(\eta-s)^{\alpha+\beta-1} t^{\alpha-1}}{P \Gamma(\alpha) \Gamma(\alpha+\beta)}, & 0 \leq s \leq t \leq 1, s \leq \eta, \\ \frac{\Gamma(\alpha+\beta)(1-s)^{\alpha-1} t^{\alpha-1}-\Gamma(\alpha) \lambda(\eta-s)^{\alpha+\beta-1} t^{\alpha-1}}{P \Gamma(\alpha) \Gamma(\alpha+\beta)}, & 0 \leq t \leq s \leq \eta \leq 1, \\ \frac{-P \Gamma(\alpha+\beta)(t-s)^{\alpha-1}+\Gamma(\alpha+\beta)(1-s)^{\alpha-1} t^{\alpha-1}}{P \Gamma(\alpha) \Gamma(\alpha+\beta)}, & 0 \leq \eta \leq s \leq t \leq 1, \\ \frac{\Gamma(\alpha+\beta)(1-s)^{\alpha-1} t^{\alpha-1}}{P \Gamma(\alpha) \Gamma(\alpha+\beta)}, & 0 \leq t \leq s \leq 1, s \geq \eta,\end{cases}
$$

with $P=1-\frac{\lambda \Gamma(\alpha)}{\Gamma(\alpha+\beta)} \eta^{\alpha+\beta-1} . G(t, s)$ is called the Green's function of boundary value problem (2.1). Obviously, $G(t, s)$ is a continuous function on $[0,1] \times[0,1]$.

Proof It is shown in $[1,2]$ that problem $(2.1)$ is equivalent to the following integral equation:

$$
u(t)=-I_{0^{+}}^{\alpha} y(t)+C_{1} t^{\alpha-1}+C_{2} t^{\alpha-2}+C_{3} t^{\alpha-3}+C_{4} t^{\alpha-4} .
$$

By $u(0)=u^{\prime}(0)=u^{\prime \prime}(0)=0$, we obtain

$$
u(t)=-I_{0^{+}}^{\alpha} y(t)+C_{1} t^{\alpha-1} .
$$

It follows from $u(1)=\lambda I_{0+}^{\beta} u(\eta)$, combined with

$$
u(1)=-I_{0^{+}}^{\alpha} y(1)+C_{1}
$$

and

$$
\lambda I_{0+}^{\beta} u(\eta)=-\lambda I_{0^{+}}^{\alpha+\beta} y(\eta)+\lambda C_{1} \frac{\Gamma(\alpha)}{\Gamma(\alpha+\beta)} \eta^{\alpha+\beta-1},
$$

that

$$
C_{1}=\frac{1}{1-\frac{\lambda \Gamma(\alpha)}{\Gamma(\alpha+\beta)} \eta^{\alpha+\beta-1}}\left\{I_{0^{+}}^{\alpha} y(1)-\lambda I_{0^{+}}^{\alpha+\beta} y(\eta)\right\}=: \frac{1}{P}\left\{I_{0^{+}}^{\alpha} y(1)-\lambda I_{0^{+}}^{\alpha+\beta} y(\eta)\right\} .
$$

Therefore, the solution to problem (2.1) is

$$
\begin{aligned}
u(t)= & -\frac{1}{\Gamma(\alpha)} \int_{0}^{t}(t-s)^{\alpha-1} y(s) \mathrm{d} s+\frac{t^{\alpha-1}}{P \Gamma(\alpha)} \int_{0}^{1}(1-s)^{\alpha-1} y(s) \mathrm{d} s \\
& -\frac{\lambda t^{\alpha-1}}{P \Gamma(\alpha+\beta)} \int_{0}^{\eta}(\eta-s)^{\alpha+\beta-1} y(s) \mathrm{d} s .
\end{aligned}
$$


For $t \leq \eta$, one has

$$
\begin{aligned}
u(t)= & -\frac{1}{\Gamma(\alpha)} \int_{0}^{t}(t-s)^{\alpha-1} y(s) \mathrm{d} s+\frac{t^{\alpha-1}}{P \Gamma(\alpha)}\left\{\int_{0}^{t}+\int_{t}^{\eta}+\int_{\eta}^{1}\right\}(1-s)^{\alpha-1} y(s) \mathrm{d} s \\
& -\frac{\lambda t^{\alpha-1}}{P \Gamma(\alpha+\beta)}\left\{\int_{0}^{t}+\int_{t}^{\eta}\right\}(\eta-s)^{\alpha+\beta-1} y(s) \mathrm{d} s \\
= & \int_{0}^{t} \frac{-P \Gamma(\alpha+\beta)(t-s)^{\alpha-1}+\Gamma(\alpha+\beta)(1-s)^{\alpha-1} t^{\alpha-1}-\Gamma(\alpha) \lambda(\eta-s)^{\alpha+\beta-1} t^{\alpha-1}}{P \Gamma(\alpha) \Gamma(\alpha+\beta)} y(s) \mathrm{d} s \\
& +\int_{t}^{\eta} \frac{\Gamma(\alpha+\beta)(1-s)^{\alpha-1} t^{\alpha-1}-\Gamma(\alpha) \lambda(\eta-s)^{\alpha+\beta-1} t^{\alpha-1}}{P \Gamma(\alpha) \Gamma(\alpha+\beta)} y(s) \mathrm{d} s \\
& +\int_{\eta}^{1} \frac{\Gamma(\alpha+\beta)(1-s)^{\alpha-1} t^{\alpha-1}}{P \Gamma(\alpha) \Gamma(\alpha+\beta) \mathrm{d} s} \\
= & \int_{0}^{1} G(t, s) y(s) \mathrm{d} s .
\end{aligned}
$$

For $t \geq \eta$, one has

$$
\begin{aligned}
u(t)= & -\frac{1}{\Gamma(\alpha)}\left\{\int_{0}^{\eta}+\int_{\eta}^{t}\right\}(t-s)^{\alpha-1} y(s) \mathrm{d} s+\frac{t^{\alpha-1}}{P \Gamma(\alpha)}\left\{\int_{0}^{\eta}+\int_{\eta}^{t}+\int_{t}^{1}\right\}(1-s)^{\alpha-1} y(s) \mathrm{d} s \\
& -\frac{\lambda t^{\alpha-1}}{P \Gamma(\alpha+\beta)} \int_{0}^{\eta}(\eta-s)^{\alpha+\beta-1} y(s) \mathrm{d} s \\
= & \int_{0}^{\eta} \frac{-P \Gamma(\alpha+\beta)(t-s)^{\alpha-1}+\Gamma(\alpha+\beta)(1-s)^{\alpha-1} t^{\alpha-1}-\Gamma(\alpha) \lambda(\eta-s)^{\alpha+\beta-1} t^{\alpha-1}}{P \Gamma(\alpha) \Gamma(\alpha+\beta)} y(s) \mathrm{d} s \\
& +\int_{\eta}^{t} \frac{-P \Gamma(\alpha+\beta)(t-s)^{\alpha-1}+\Gamma(\alpha+\beta)(1-s)^{\alpha-1} t^{\alpha-1}}{P \Gamma(\alpha) \Gamma(\alpha+\beta) \mathrm{d} s} \\
& +\int_{t}^{1} \frac{\Gamma(\alpha+\beta)(1-s)^{\alpha-1} t^{\alpha-1}}{P \Gamma(\alpha) \Gamma(\alpha+\beta)} y(s) \mathrm{d} s \\
= & \int_{0}^{1} G(t, s) y(s) \mathrm{d} s .
\end{aligned}
$$

The proof is finished.

A careful analysis of the Green's function allows us to deduce the following results.

Lemma 2.2 The Green's function $G(t, s)$ has the following properties:

(1) $G(t, s)>0, \forall t, s \in(0,1)$;

(2) $G(1, s)>0, \forall s \in(0,1)$;

(3) $G(t, s) \leq \frac{(1-s)^{\alpha-1} t^{\alpha-1}}{P \Gamma(\alpha)}, \forall t, s \in(0,1)$;

(4) $G(t, s) \geq \frac{\lambda t^{\alpha-1} \eta^{\alpha+\beta-1}}{P \Gamma(\alpha+\beta)}\left\{(1-s)^{\alpha-1}-(1-s)^{\alpha+\beta-1}\right\}, \forall t, s \in(0,1)$.

Proof Assume at first that $0 \leq s \leq t \leq 1, s \leq \eta, 0 \leq \frac{\lambda \Gamma(\alpha) \eta^{\alpha+\beta-1}}{\Gamma(\alpha+\beta)}<1$, then we have

$$
P \Gamma(\alpha) \Gamma(\alpha+\beta) G(t, s)
$$

$$
=-P \Gamma(\alpha+\beta)(t-s)^{\alpha-1}+\Gamma(\alpha+\beta)(1-s)^{\alpha-1} t^{\alpha-1}-\Gamma(\alpha) \lambda(\eta-s)^{\alpha+\beta-1} t^{\alpha-1}
$$




$$
\begin{aligned}
= & \lambda \Gamma(\alpha) \eta^{\alpha+\beta-1}(t-s)^{\alpha-1}+\left\{-\Gamma(\alpha+\beta)(t-s)^{\alpha-1}+\Gamma(\alpha+\beta)(1-s)^{\alpha-1} t^{\alpha-1}\right\} \\
& -\Gamma(\alpha) \lambda(\eta-s)^{\alpha+\beta-1} t^{\alpha-1} \\
\geq & \lambda \Gamma(\alpha) \eta^{\alpha+\beta-1}(t-s)^{\alpha-1}-\lambda \Gamma(\alpha) \eta^{\alpha+\beta-1}(t-s)^{\alpha-1} \\
& +\lambda \Gamma(\alpha) \eta^{\alpha+\beta-1}(1-s)^{\alpha-1} t^{\alpha-1}-\Gamma(\alpha) \lambda(\eta-s)^{\alpha+\beta-1} t^{\alpha-1} \\
= & \lambda \Gamma(\alpha) \eta^{\alpha+\beta-1}(1-s)^{\alpha-1} t^{\alpha-1}-\Gamma(\alpha) \lambda(\eta-s)^{\alpha+\beta-1} t^{\alpha-1} \\
\geq & \lambda \Gamma(\alpha) \eta^{\alpha+\beta-1} t^{\alpha-1}\left\{(1-s)^{\alpha-1}-(1-s)^{\alpha+\beta-1}\right\}
\end{aligned}
$$

and

$$
\begin{aligned}
& P \Gamma(\alpha) \Gamma(\alpha+\beta) G(t, s) \\
&=-P \Gamma(\alpha+\beta)(t-s)^{\alpha-1}+\Gamma(\alpha+\beta)(1-s)^{\alpha-1} t^{\alpha-1}-\Gamma(\alpha) \lambda(\eta-s)^{\alpha+\beta-1} t^{\alpha-1} \\
&=\lambda \Gamma(\alpha) \eta^{\alpha+\beta-1}(t-s)^{\alpha-1}-\Gamma(\alpha+\beta)(t-s)^{\alpha-1}+\Gamma(\alpha+\beta)(1-s)^{\alpha-1} t^{\alpha-1} \\
& \quad-\Gamma(\alpha) \lambda(\eta-s)^{\alpha+\beta-1} t^{\alpha-1} \\
& \leq \Gamma(\alpha+\beta)(1-s)^{\alpha-1} t^{\alpha-1}-\Gamma(\alpha) \lambda(\eta-s)^{\alpha+\beta-1} t^{\alpha-1} \\
& \leq \Gamma(\alpha+\beta)(1-s)^{\alpha-1} t^{\alpha-1} .
\end{aligned}
$$

For $0 \leq t \leq s \leq \eta \leq 1$, we have

$$
\begin{aligned}
P & \Gamma(\alpha) \Gamma(\alpha+\beta) G(t, s) \\
& =\Gamma(\alpha+\beta)(1-s)^{\alpha-1} t^{\alpha-1}-\Gamma(\alpha) \lambda(\eta-s)^{\alpha+\beta-1} t^{\alpha-1} \\
& \geq \lambda \Gamma(\alpha) \eta^{\alpha+\beta-1}(1-s)^{\alpha-1} t^{\alpha-1}-\Gamma(\alpha) \lambda(\eta-s)^{\alpha+\beta-1} t^{\alpha-1} \\
& \geq \lambda \Gamma(\alpha) \eta^{\alpha+\beta-1} t^{\alpha-1}\left\{(1-s)^{\alpha-1}-(1-s)^{\alpha+\beta-1}\right\}
\end{aligned}
$$

and

$$
\begin{aligned}
P & \Gamma(\alpha) \Gamma(\alpha+\beta) G(t, s) \\
& =\Gamma(\alpha+\beta)(1-s)^{\alpha-1} t^{\alpha-1}-\Gamma(\alpha) \lambda(\eta-s)^{\alpha+\beta-1} t^{\alpha-1} \\
& \leq \Gamma(\alpha+\beta)(1-s)^{\alpha-1} t^{\alpha-1} .
\end{aligned}
$$

For $0 \leq \eta \leq s \leq t \leq 1$, we have

$$
\begin{aligned}
P \Gamma(\alpha) \Gamma(\alpha+\beta) G(t, s) \\
=-P \Gamma(\alpha+\beta)(t-s)^{\alpha-1}+\Gamma(\alpha+\beta)(1-s)^{\alpha-1} t^{\alpha-1} \\
=\lambda \Gamma(\alpha) \eta^{\alpha+\beta-1}(t-s)^{\alpha-1}-\Gamma(\alpha+\beta)(t-s)^{\alpha-1}+\Gamma(\alpha+\beta)(1-s)^{\alpha-1} t^{\alpha-1} \\
\geq \lambda \Gamma(\alpha) \eta^{\alpha+\beta-1}(t-s)^{\alpha-1}-\lambda \Gamma(\alpha) \eta^{\alpha+\beta-1}(t-s)^{\alpha-1} \\
\quad+\lambda \Gamma(\alpha) \eta^{\alpha+\beta-1}(1-s)^{\alpha-1} t^{\alpha-1} \\
\geq \lambda \Gamma(\alpha) \eta^{\alpha+\beta-1} t^{\alpha-1}\left\{(1-s)^{\alpha-1}-(1-s)^{\alpha+\beta-1}\right\}
\end{aligned}
$$


and

$$
\begin{aligned}
P & \Gamma(\alpha) \Gamma(\alpha+\beta) G(t, s) \\
& =-P \Gamma(\alpha+\beta)(t-s)^{\alpha-1}+\Gamma(\alpha+\beta)(1-s)^{\alpha-1} t^{\alpha-1} \\
& =\lambda \Gamma(\alpha) \eta^{\alpha+\beta-1}(t-s)^{\alpha-1}-\Gamma(\alpha+\beta)(t-s)^{\alpha-1}+\Gamma(\alpha+\beta)(1-s)^{\alpha-1} t^{\alpha-1} \\
& \leq \Gamma(\alpha+\beta)(1-s)^{\alpha-1} t^{\alpha-1} .
\end{aligned}
$$

For $0 \leq t \leq s \leq 1, s \geq \eta$, we have

$$
\begin{aligned}
P & \Gamma(\alpha) \Gamma(\alpha+\beta) G(t, s) \\
& =\Gamma(\alpha+\beta)(1-s)^{\alpha-1} t^{\alpha-1} \\
& \geq \lambda \Gamma(\alpha) \eta^{\alpha+\beta-1} t^{\alpha-1}\left\{(1-s)^{\alpha-1}-(1-s)^{\alpha+\beta-1}\right\} .
\end{aligned}
$$

From the above, (3), (4) are complete. Clearly, (1) and (2) are true. The proof is completed.

From Lemma 2.2, we illustrate the following lemma without proof.

Lemma 2.3 The Green's function $G(t, s)$ satisfies

$$
t^{\alpha-1} w_{1}(s) \leq G(t, s) \leq t^{\alpha-1} w_{2}(s), \quad \forall t, s \in(0,1)
$$

where

$$
w_{1}(s)=\frac{\lambda \eta^{\alpha+\beta-1}}{P \Gamma(\alpha+\beta)}\left\{(1-s)^{\alpha-1}-(1-s)^{\alpha+\beta-1}\right\}, \quad w_{2}(s)=\frac{(1-s)^{\alpha-1}}{P \Gamma(\alpha)}
$$

\section{Main results}

Let Banach space $E=C([0,1])$ be endowed with the norm $\|u\|_{\infty}=\max _{0 \leq t \leq 1}|u(t)|$. A closed cone $K \subset E$ by $K=\{u \in E: u \succeq 0\}$, where 0 is the zero function, and the cone $K$ is normal. Set $K_{a}=\{u \in K:\|u\| \leq a\}$. Define the operator $T: K_{a} \rightarrow E$ as

$$
(T u)(t)=\int_{0}^{1} G(t, s) f(s, u(s)) \mathrm{d} s, \quad t \in[0,1]
$$

It is not hard to see that the fixed points of operator $T$ coincide with the solutions to the problem (1.1).

Lemma 3.1 ([26]) Let $X$ be a Banach space ordered by a normal cone $K \subset X$. Assume that $T:\left[x_{1}, x_{2}\right] \rightarrow X$ is a completely continuous increasing operator such that $x_{1} \preceq T x_{1}$, $x_{2} \succeq T x_{2}$. Then $T$ has a minimal fixed point $x_{*}$ and a maximal fixed point $x^{*}$ such that $x_{1} \preceq x_{*} \preceq x^{*} \preceq x_{2}$. Moreover, $x_{*}=\lim _{n \rightarrow \infty} T^{n} x_{1}, x^{*}=\lim _{n \rightarrow \infty} T^{n} x_{2}$, where $\left\{T^{n} x_{1}\right\}_{n=1}^{\infty}$ is an increasing sequence, $\left\{T^{n} x_{2}\right\}_{n=1}^{\infty}$ is a decreasing sequence.

For the forthcoming analysis, we need the following assumptions: 
$\left(\mathrm{A}_{1}\right) f:[0,1] \times[0, a] \rightarrow[0, \infty)$ is continuous and $f(t, 0) \not \equiv 0$;

$\left(\mathrm{A}_{2}\right)$ there exists a nonnegative function $j \in C[0,1] \subseteq L^{1}[0,1]$ such that $|f(t, u)| \leq j(t)$, $(t, u) \in[0,1] \times[0, a]$

$\left(\mathrm{A}_{3}\right) f(t, \underline{u}) \leq f(t, \bar{u}), t \in[0,1], 0 \leq \underline{u} \leq \bar{u} \leq a$.

Lemma 3.2 Assume that $\left(\mathrm{A}_{1}\right)-\left(\mathrm{A}_{3}\right)$ hold, then the operator $T$ defined in (3.1) is a completely continuous increasing operator.

Proof Firstly, the operator $T$ is continuous in view of the continuity of functions $f(t, u(t))$ and $G(t, s)$.

Secondly, we will show that $T\left(K_{a}\right)$ is bounded. Let

$$
L=\int_{0}^{1} j(t) \mathrm{d} t<\infty
$$

Then, for any $u \in K_{a}$, we have

$$
\|T u(t)\|=\max _{t \in[0,1]} \int_{0}^{1} G(t, s)|f(s, u(s))| \mathrm{d} s \leq \frac{L}{P \Gamma(\alpha)}, \quad t \in[0,1] .
$$

For each $u \in K_{a}$, one can show that

$$
\begin{aligned}
\left|(T u)^{\prime}(t)\right|=\mid & -\frac{1}{\Gamma(\alpha-1)} \int_{0}^{t}(t-s)^{\alpha-2} f(s, u(s)) \mathrm{d} s \\
& +\frac{t^{\alpha-2}}{P \Gamma(\alpha-1)} \int_{0}^{1}(1-s)^{\alpha-1} f(s, u(s)) \mathrm{d} s \\
& -\frac{(\alpha-1) \lambda t^{\alpha-2}}{P \Gamma(\alpha+\beta)} \int_{0}^{\eta}(\eta-s)^{\alpha+\beta-1} f(s, u(s)) \mathrm{d} s \mid \\
\leq & \frac{1}{\Gamma(\alpha-1)} \int_{0}^{t}(t-s)^{\alpha-2}|f(s, u(s))| \mathrm{d} s \\
& +\frac{t^{\alpha-2}}{P \Gamma(\alpha-1)} \int_{0}^{1}(1-s)^{\alpha-1}|f(s, u(s))| \mathrm{d} s \\
& +\frac{(\alpha-1) \lambda t^{\alpha-2}}{P \Gamma(\alpha+\beta)} \int_{0}^{\eta}(\eta-s)^{\alpha+\beta-1}|f(s, u(s))| \mathrm{d} s \\
\leq & \frac{L}{\Gamma(\alpha-1)}+\frac{L}{P \Gamma(\alpha-1)}+\frac{(\alpha-1) \lambda L}{P \Gamma(\alpha+\beta)}:=\bar{L} .
\end{aligned}
$$

Therefore, for any $t_{1}, t_{2} \in[0,1]$ with $t_{1}<t_{2}$, we have

$$
\left|(T u)\left(t_{2}\right)-(T u)\left(t_{1}\right)\right| \leq \int_{t_{1}}^{t_{2}}\left|(T u)^{\prime}(s)\right| \mathrm{d} s \leq \bar{L}\left(t_{2}-t_{1}\right)
$$

that is, $T\left(K_{a}\right)$ is equicontinuous.

The Arzela-Ascoli theorem implies that the operator $T: K_{a} \rightarrow E$ is completely continuous.

The assumption $\left(\mathrm{A}_{3}\right)$ provides that the operator $T: K_{a} \rightarrow E$ is an increasing operator. The proof is completed. 
Theorem 3.1 Assume that $\left(\mathrm{A}_{1}\right)-\left(\mathrm{A}_{3}\right)$ hold, and

$$
\int_{0}^{1} w_{1}(s) f(s, 0) \mathrm{d} s \geq 0, \quad \int_{0}^{1} w_{2}(s) f\left(s, a s^{\alpha-1}\right) \mathrm{d} s \leq a, \quad s \in[0,1],
$$

then the problem (1.1) has two positive solutions $u^{*}, v^{*}$ satisfying $0<u^{*} \leq v^{*} \leq a$. Moreover, there exist a non-decreasing iterative sequence $\left\{u_{n}\right\}_{n=0}^{\infty}$ with

$$
\lim _{n \rightarrow \infty} u_{n}=u^{*}, \quad u_{0}=0, \quad u_{n+1}=T u_{n}, \quad n=0,1,2, \ldots
$$

and a non-increasing iterative sequence $\left\{v_{n}\right\}_{n=0}^{\infty}$ with

$$
\lim _{n \rightarrow \infty} v_{n}=v^{*}, \quad v_{0}=a t^{\alpha-1}, \quad v_{n+1}=T v_{n}, \quad n=0,1,2, \ldots, t \in[0,1]
$$

Proof We only need to prove that $T u_{0} \geq u_{0}$ and $T v_{0} \leq v_{0}$ :

$$
\begin{aligned}
T u_{0} & =\int_{0}^{1} G(t, s) f\left(s, u_{0}\right) \mathrm{d} s=\int_{0}^{1} G(t, s) f(s, 0) \mathrm{d} s \\
& \geq t^{\alpha-1} \int_{0}^{1} w_{1}(s) f(s, 0) \mathrm{d} s \geq 0=u_{0}, \quad t \in[0,1],
\end{aligned}
$$

implies $u_{1} \geq u_{0}$, which combined with $\left(\mathrm{A}_{3}\right)$ gives

$$
\begin{aligned}
u_{2} & =T u_{1}=\int_{0}^{1} G(t, s) f\left(s, u_{1}\right) \mathrm{d} s \\
& \geq \int_{0}^{1} G(t, s) f\left(s, u_{0}\right) \mathrm{d} s=u_{1}, \quad t \in[0,1] .
\end{aligned}
$$

Similarly, we have

$$
\begin{aligned}
v_{1} & =T v_{0}=\int_{0}^{1} G(t, s) f\left(s, v_{0}\right) \mathrm{d} s \\
& \leq t^{\alpha-1} \int_{0}^{1} w_{2}(s) f\left(s, a t^{\alpha-1}\right) \mathrm{d} s \\
& \leq a t^{\alpha-1}=v_{0}, \quad t \in[0,1] .
\end{aligned}
$$

By induction, one can prove that $u_{n+1} \geq u_{n}$ and $v_{n+1} \leq u_{n}$.

Lemma 3.1 shows that the operator $T$ has a minimal fixed point $u^{*}$ and a maximal fixed point $v^{*}$ satisfying $0 \leq u^{*} \leq v^{*} \leq a$.

From $\left(\mathrm{A}_{1}\right)$, we find that the zero function is not the solution to the problem (1.1). Thus $0<u^{*} \leq v^{*} \leq a$. The proof is finished.

Remark 3.1 The iterative sequences in Theorem 3.1 starting with a simple function is helpful for calculating.

\section{An example}

Consider the following boundary value problem:

$$
\left\{\begin{array}{l}
D_{0+}^{7 / 2} u(t)+100 t^{5}+0.001+\frac{\sin t}{400}-u^{2}(t)+20 u(t)=0, \quad 0<t<1, \\
u(0)=u^{\prime}(0)=u^{\prime \prime}(0)=0, \quad u(1)=5 I_{0+}^{3 / 2} u\left(\frac{1}{2}\right),
\end{array}\right.
$$


where $\alpha=\frac{7}{2}, \beta=\frac{3}{2}, \eta=\frac{1}{2}, \lambda=5,0 \leq \frac{\lambda \Gamma(\alpha) \eta^{\alpha+\beta-1}}{\Gamma(\alpha+\beta)} \approx 0.0433<1$, and

$$
f(t, u(t))=100 t^{5}+\frac{\sin t}{400}+0.001-u^{2}(t)+20 u(t)
$$

We take $a=10$ for calculating conveniently. Then the assumptions $\left(\mathrm{A}_{1}\right)-\left(\mathrm{A}_{3}\right)$ hold, and

$$
\begin{aligned}
& f(t, 0)=0.001+100 t^{5}+\frac{\sin t}{400} \\
& f\left(t, 10 t^{5 / 2}\right)=\frac{\sin t}{400}+200 t^{5 / 2}+0.001
\end{aligned}
$$

A simple calculation leads to

$$
\begin{aligned}
& w_{1}(s)=0.0136\left\{(1-s)^{5 / 2}-(1-s)^{4}\right\}, \\
& w_{2}(s)=0.3146(1-s)^{5 / 2}, \\
& \int_{0}^{1} w_{1}(s) f(s, 0) \mathrm{d} s \approx 0.0033589 \geq 0, \\
& \int_{0}^{1} w_{2}(s) f\left(s, a s^{\alpha-1}\right) \mathrm{d} s \approx 0.965319 \leq 10 .
\end{aligned}
$$

By Theorem 3.1, the problem (4.1) has two nontrivial solutions $u^{*}, v^{*}$ with $0<u^{*} \leq v^{*} \leq$ 10 , and the two monotone iterative sequences $\left\{u_{n}\right\}_{n=1}^{\infty}$ and $\left\{v_{n}\right\}_{n=1}^{\infty}$ can be taken as

$$
u_{0}=0, \quad u_{n+1}=T u_{n}, \quad v_{0}=10 t^{\alpha-1}, \quad v_{n+1}=T v_{n}, \quad n=0,1,2, \ldots
$$

Using MATLAB, the iterative sequences are computed and are depicted in Figure 1 and Figure 2 .

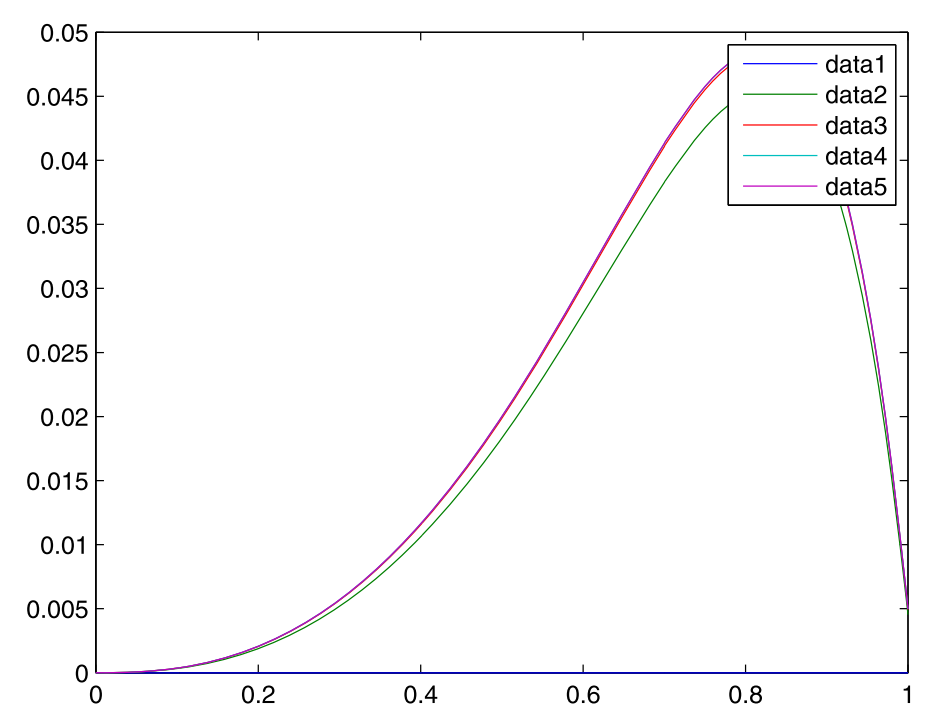

Figure 1 The non-decreasing sequence $\left\{u_{n}(t)\right\}_{n=0}^{4}$ in the interval $[0,1]$. 


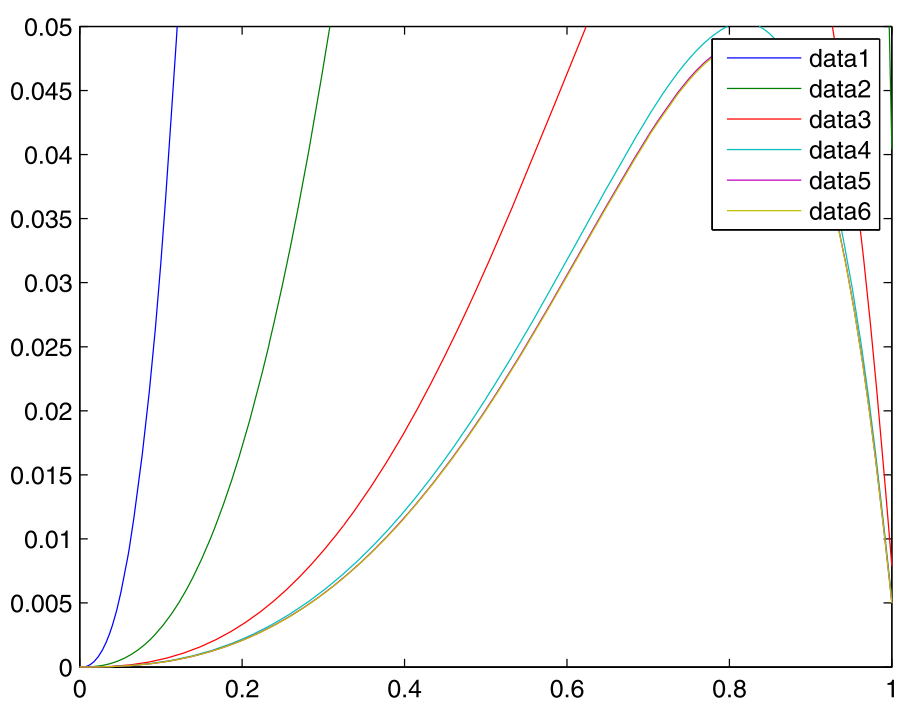

Figure 2 The non-increasing sequence $\left\{v_{n}(t)\right\}_{n=0}^{5}$ in the interval $[0,1]$.

\section{Competing interests}

The authors declare that they have no competing interests.

\section{Authors' contributions}

All authors contributed equally to the manuscript. All authors read and approved the final manuscript.

\section{Author details}

${ }^{1}$ School of Mathematics, Jilin University, Changchun, 130012, P.R. China. ${ }^{2}$ College of Science, Changchun University of Science and Technology, Changchun, 130022, P.R. China.

\section{Acknowledgements}

The authors are grateful to anonymous referees for their constructive comments and suggestions which have greatly improved this paper. This work is supported by NSF of China (11271154).

Received: 15 January 2015 Accepted: 7 May 2015 Published online: 18 June 2015

\section{References}

1. Samko, SG, Kilbas, AA, Marichev, Ol: Fractional Integral and Derivatives: Theory and Applications. Gordon \& Breach, Yverdon (1993)

2. Miller, KS, Ross, B: An Introduction to the Fractional Calculus and Fractional Differential Equations. Wiley, New York (1993)

3. Podlubny, I: Fractional Differential Equations. Academic Press, San Diego (1999)

4. Kilbas, AA, Trujilo, JJ: Differential equations of fractional order: methods, results and problems I. Appl. Anal. 78 153-192 (2001)

5. Kilbas, AA, Srivastava, HM, Trujillo, JJ: Theory and Applications of Fractional Differential Equations. North-Holland Mathematics Studies, vol. 204. Elsevier, Amsterdam (2006)

6. Sabatier, J, Agrawal, OP, Machado, J: Advances in Fractional Calculus: Theoretical Developments and Applications in Physics and Engineering. Springer, Dordrecht (2007)

7. Lakshmikantham, V, Vatsala, AS: Basic theory of fractional differential equations. Nonlinear Anal. 69(8), 2677-2682 (2008)

8. Bai, ZB: On positive solutions of a nonlocal fractional boundary value problem. Nonlinear Anal. 72, 916-924 (2010)

9. Bai, ZB: The method of lower and upper solutions for a bending of an elastic beam equation. J. Math. Anal. Appl. 248 195-202 (2000)

10. Bai, ZB, Sun, WC: Existence of solutions for a fractional multi-point boundary value problem. Comput. Math. Appl. 63, 1369-1381 (2012)

11. Benchohra, M, Graef, JR, Hamani, S: Existence results for boundary value problems with nonlinear fractional differential equations. Appl. Anal. 87, 851-863 (2008)

12. Han, $\mathrm{XL}, \mathrm{Gao}, \mathrm{HL}$ : Existence of positive solutions for eigenvalue problem of nonlinear fractional differential equations. Adv. Differ. Equ. 2012, 66 (2012)

13. Chen, $\mathrm{Y}$, Tang, $\mathrm{XH}$ : Positive solutions of fractional differential equations at resonance on the half-line. Bound. Value Probl. 2012, 64 (2012)

14. Alsaedi, A, Ahmad, B, Assolami, A: On antiperiodic boundary value problems for higher-order fractional differential equations. Abstr. Appl. Anal. 2012, Article ID 325984 (2012)

15. Bai, ZB, Sun, WC, Zhang, W: Positive solutions for boundary value problems of singular fractional differential equations. Abstr. Appl. Anal. 2013, Article ID 129640 (2013) 
16. Zhang, HN, Gao, WJ: Existence and uniqueness results for a coupled system of nonlinear fractional equations with antiperiodic boundary conditions. Abstr. Appl. Anal. 2014, Article ID 463517 (2014)

17. Al-Refai, M, Hajji, M: Monotone iterative sequences for nonlinear boundary value problems of fractional order Nonlinear Anal. 74, 3531-3539 (2011)

18. Sun, B, Yang, A, Ge, W: Successive iteration and positive solutions for some second-order three-point $p$-Laplacian boundary value problems. Math. Comput. Model. 50, 344-350 (2009)

19. Zhang, $X, L i u, L, W u, Y, L u, Y: T h e$ iterative solutions of nonlinear fractional differential equations. Appl. Math. Comput. 219, 4680-4691 (2013)

20. Ahmad, B, Nieto, JJ: Riemann-Liouville fractional integro-differential equations with fractional nonlocal integral boundary conditions. Bound. Value Probl. 2011, 36 (2011)

21. Ahmad, B, Agarwal, RP: Some new versions of fractional boundary value problems with slit-strips conditions. Bound Value Probl. 2014, 175 (2014)

22. Zhang, L, Ahmad, B, Wang, G: Successive iterations for positive extremal solutions of nonlinear fractional differential equations on a half-line. Bull. Aust. Math. Soc. 91(1), 116-128 (2015)

23. Zhang, $X$, Wang, L, Sun, Q: Existence of positive solutions for a class of nonlinear fractional differential equations with integral boundary conditions and a parameter. Appl. Math. Comput. 226, 708-718 (2014)

24. Jiang, M, Zhong, S: Successively iterative method for fractional differential equations with integral boundary conditions. Appl. Math. Lett. 38, $94-99$ (2014)

25. Sun, Y, Zhao, M: Positive solutions for a class of fractional differential equations with integral boundary conditions. Appl. Math. Lett. 34, 17-21 (2014)

26. Amann, H: Fixed point equations and nonlinear eigenvalue problems in order Banach spaces. SIAM Rev. 18, 620-709 (1976)

\section{Submit your manuscript to a SpringerOpen ${ }^{\mathcal{O}}$ journal and benefit from:}

- Convenient online submission

- Rigorous peer review

- Immediate publication on acceptance

- Open access: articles freely available online

- High visibility within the field

- Retaining the copyright to your article 